\title{
Ancient and modern hybridization between Lucilia sericata and $L$. cuprina (Diptera: Calliphoridae)
}

\author{
KIRSTIN WILLIAMS ${ }^{1,2}$ and MARTIN H. VILLET ${ }^{2}$ \\ ${ }^{1}$ Durban Natural Science Museum, PO Box 4085, Durban, 4000, South Africa; e-mail: WilliamsK@durban.gov.za \\ ${ }^{2}$ Southern African Forensic Entomology Research Laboratory, Department of Zoology and Entomology, Rhodes University, \\ PO Box 94, Grahamstown, 6140, South Africa; e-mail: M.Villet@ru.ac.za
}

Key words. Diptera, Calliphoridae, Lucilia sericata, L. cuprina, hybrids, mDNA, phylogenetic, taxonomy, introgression

\begin{abstract}
There are important but inconsistent differences in breeding site preference between the blow flies Lucilia sericata (Meigen, 1826) and L. cuprina (Wiedemann, 1830) (Diptera: Calliphoridae) that have significance for medical and veterinary science. These inconsistencies might arise from hybridisation. The species are difficult to distinguish using external morphology, although the male genitalia are distinctive and there are reliable molecular markers. Molecular evidence of modern hybridisation, derived from a newly developed nuclear marker, the period (per) gene, is presented here. This has implications for identifications of these species based on mtDNA, and may lead to an explanation of the medical and veterinary anomalies noted in these species.
\end{abstract}

\section{INTRODUCTION}

The use of Lucilia blowflies for maggot debridement therapy (MDT) has become a topic of great interest in South Africa (Williams et al., 2008; F. Cronje \& Du Plessis H.J.C, pers. comm). Lucilia sericata (Meigen, 1826 ) is the species of choice for MDT (Altincicek \& Vilcinskas, 2009; Vilcinskas, 2011), but the misidentification of Lucilia cuprina (Wiedemann, 1830) and L. sericata for use in MDT and how best to supplement MDT colonies has raised the issue of species identification (Williams et al., 2008; Tantawi et al., 2010). Lucilia cuprina has recently been used successfully for MDT (Paul et al., 2009; Tantawi et al., 2010; Kingu et al., 2012) although this species is responsible for sheep-strike that causes losses to the wool and meat industries that amount to millions of dollars worldwide each year (Hepburn, 1943; Ullyett, 1945; Vogt \& Woodburn, 1979; Heath \& Bishop, 2006). Different populations of L. sericata show different degrees of cuprina-like attraction to sheep (Crombe, 1944; Cragg, 1956), but no clear pattern in this myiasis has been noted.

These two species have been suspected of interbreeding and producing fertile hybrids in South Africa (Ullyett, 1945). They have been shown to hybridise under laboratory conditions and to produce fertile hybrids, although there are no reports of this occurring naturally (Ullyett, 1945). Lucilia cuprina has consistently been found to be paraphyletic relative to $L$. sericata in studies of several mitochondrial genes (Table 1). If they are interbreeding, this leads to an explanation of the medical and veterinary anomalies noted in the biology of these species.

Several authors have suggested that these flies should be classified as three species or that L cuprina should be classified as two subspecies - Lucilia c. cuprina (Wiedemann, 1830) and L. c. dorsalis Robineau-Desvoidy, 1830 (Waterhouse \& Paramonov, 1950; Norris, 1990; Stevens
\& Wall, 1996; Stevens et al., 2002; Stevens, 2003; Wallman et al., 2005; Wells et al., 2007; DeBry et al., 2010). Lucilia sericata and L. cuprina are morphologically very similar and the adults are difficult to identify using the available keys based on morphological characters without using the male genitalia, which usually requires destructive sampling (Aubertin, 1933; Smith, 1986; Norris, 1990; Holloway, 1991). However, with some experience, the females can usually be reliably identified using the characteristics described by Holloway (1991a).

Molecular methods are useful in confirming the taxonomic status of these two species (Williams et al., 2008; Tourle et al., 2009; Tantawi et al., 2010). The use of more than one gene for taxonomic and phylogenetic studies is recommended as using only one gene may not give a true picture of relationships or patterns of gene flow (Sperling et al., 1994; Nelson et al., 2007; Whitworth et al., 2007; Tourle et al., 2009). Analysing both nuclear and mitochondrial genes simultaneously has highlighted introgression and the difference between gene trees and species trees (Page \& Charleston, 1997; Nichols, 2001; Stevens et al., 2002; Stevens, 2003; Whitworth et al., 2007; Tourle et al., 2009; DeBry et al., 2010).

The purpose of this study was to test for evidence of hybridisation between these two species, shown by a difference between the gene trees produced from sequence data using nuclear, as opposed to mitochondrial, genes from these flies from different localities around South Africa and from sites in Africa, Europe, Australia, Asia, and North America.

\section{MATERIAL AND METHODS}

Adult flies of both L. sericata and L. cuprina were collected in Britstown, Bloemfontein, Cape Town, Durban, Grahamstown, Nelspruit, and Witbank in South Africa (Fig. 1 insert). Lucilia specimens originating from Welkom and Pretoria were 


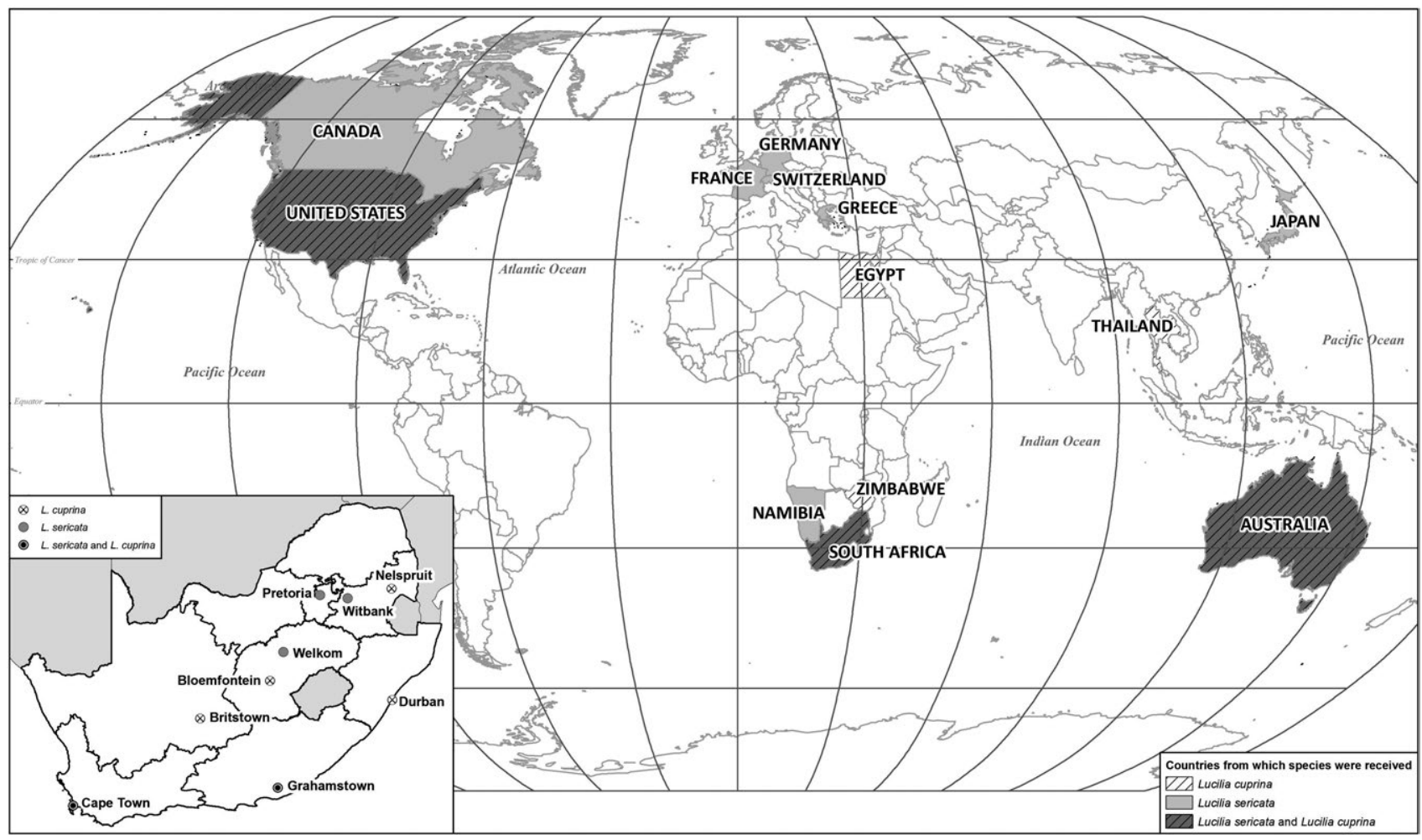

Fig. 1. World map showing the localities where flies were caught. Insert: map of South Africa showing the towns where flies were caught.

also obtained from a maggot debridement therapy colony at Eugene Marais Hospital in Pretoria. Lucilia sericata was also obtained from Australia, Canada, France, Germany, Greece, Japan, Namibia, Switzerland, and the United States of America (Fig. 1). Additional specimens of L. cuprina were obtained from Australia, Egypt, Thailand, the United States of America, and Zimbabwe (Fig. 1). A total of 84 flies were collected -11 males and 73 females. They were identified by their morphology using published keys (Aubertin, 1933; Smith, 1986; Holloway, 1991a). Due to the biology of these flies, females are attracted to bait traps more than males and therefore characteristics identified by Holloway (1991a); specifically the distances and angles between setae on the vertex of females, the extent of metallic sheen on the parafrontal sclerites of females and the number of scutellar setulae were used to identify these flies.

All flies were kept in separate $1.5 \mathrm{ml}$ Eppendorf tubes in $96 \%$ ethanol and deposited with the Durban Natural Science Museum after analysis. One hind leg of each fly was used for DNA analysis. DNA was extracted using the Qiagen DNeasy tissue kit (Qiagen, Inc., Valencia, CA) according to the manufacturer's instructions (Qiagen 07/2006).

Three genes were chosen for sequencing - $28 S$ rRNA (28S), a nuclear gene that has been used in previous studies and would allow comparison with other studies (Table 1); period (per), a second nuclear gene that is faster-evolving than $28 S$ to give better resolution; and cytochrome oxidase I (COI), that has been used in previous studies (Table 1). A region of approximately $650 \mathrm{bp}$ in domain $1-2$ of the $28 S$ gene was amplified using the primers 5'-CCCCCTGAATTTAAGCATAT-3' and 5'GTTAGACTCCTTGGTCCGTG-3' (Stevens et al., 2002). A region of approximately $600 \mathrm{bp}$ of the $C O I$ gene was amplified using the primers C1-J1709 (5'-AATTGGGGGGTTTGGAA ATTG-3') and C1-N2353 (5'-GCTCGTGTATCAACGTCTA TTCC-3') (Simon et al., 2006). This region overlaps the "barcoding" region for approximately 300 base pairs. A region of approximately $730 \mathrm{bp}$ of the per gene, was amplified using the primers per5 (5'-GCCTTCAGATACGGTCAAAC-3') (G. Warman, pers. comm.) and per reverse (5'-CCGAGTGTGGTTTG

TABLE 1. Genes used in studies of Lucilia sericata and Lucilia cuprina.

\begin{tabular}{|c|c|c|c|c|c|}
\hline \multirow{2}{*}{ Source } & \multicolumn{2}{|l|}{ Mitochondrial } & \multicolumn{3}{|c|}{ Nuclear } \\
\hline & $\mathrm{CO1}$ & 12S rRNA & $28 S$ rRNA & per & RAPDs \\
\hline Stevens \& Wall, 1996 & - & 329 bp & - & - & $\mathrm{X}$ \\
\hline Stevens et al., 2002 & 2300 bp (CO1 \& 2) & - & $2193 \mathrm{bp}$ & - & \\
\hline Stevens, 2003 & 2300 bp (CO1 \& 2) & - & $2200 \mathrm{bp}$ & - & \\
\hline Wallman et al., 2005 & 3008 bp $(C O 1 \& 2 \& N D 4-N D 4 L)$ & - & - & - & \\
\hline Wells et al., 2007 & $1545 \mathrm{bp}$ & - & - & - & \\
\hline Harvey et al., 2008 & $1167 \mathrm{bp}$ & - & - & - & \\
\hline Williams et al., 2008 & $601 \mathrm{bp}$ & - & $654 \mathrm{bp}$ & - & \\
\hline Tourle et al., 2009 & $439 \mathrm{bp}$ & - & $678 \mathrm{bp}$ & - & \\
\hline DeBry et al., 2010 & $1200 \mathrm{bp}$ & - & $2100 \mathrm{bp}$ & - & \\
\hline Tantawi et al., 2010 & $576 \mathrm{bp}$ & - & $656 \mathrm{bp}$ & 746 bp & \\
\hline This study & $576 \mathrm{bp}$ & - & $654 \mathrm{bp}$ & 722 bp & \\
\hline
\end{tabular}


TABLE 2. Specimen locality data for sequences included from GenBank.

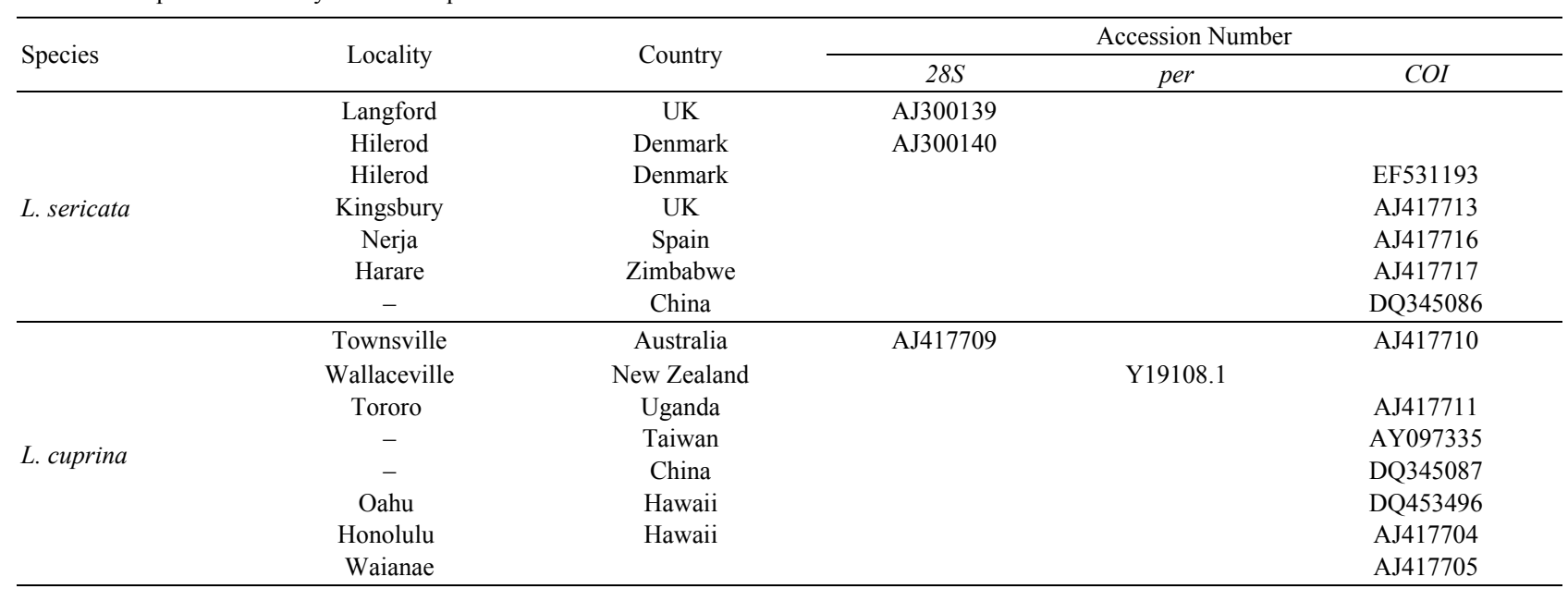

GAGATT-3') (designed by the authors). Polymerase chain reaction (PCR) amplification was performed using $1 \mu \mathrm{L}$ of DNA in a $25 \mu \mathrm{L}$ reaction. Amplification times were $94^{\circ} \mathrm{C}$ for $5 \mathrm{~min}$ denaturation, followed by 36 cycles of $94^{\circ} \mathrm{C}$ for $30 \mathrm{~s}, 55^{\circ} \mathrm{C}$ for 1 min, $72^{\circ} \mathrm{C}$ for $30 \mathrm{~s}$ and a final extension period at $72^{\circ} \mathrm{C}$ for 7 min. PCR products were confirmed by gel electrophoresis stained in ethidium bromide.

PCR products were then sequenced using an ABI 37301 Genetic Analyzer (Applied Biosystems, Foster City, CA, USA) and the primers used in amplification. Additional DNA sequences for these two species were obtained from GenBank (www.ncbi.nlm.nih.gov) for comparative analysis (Table 2). The sequences were aligned and edited using the BioEdit v7.0.9 software (Hall, 1999).

Phylogenetic reconstruction by maximum parsimony analysis was performed using PAUP*4b10 (Swofford, 2003) using the best-fitting model (HKY) from MrModelTest v2.2 (Nylander, 2004) applied in MrMTgui (Nuin, 2005). Statistical support for nodes was assessed by bootstrapping with 100 replicates retaining a maximum of 10,000 trees. Bayesian inference analysis was performed using one cold and three hot chains and the HKY model. Analysis was run for 5,000,000 generations, sampling every 1,000 generations with burn-in of 1,000 samples. All phylogenetic analyses used Calliphora vicina and Lucilia infernalis as outgroups. Incongruence length difference (ILD) tests (Farris et al., 1994) were run in PAUP* 4 b10 (Swofford, 2003) to quantify the differences in topology between trees for $28 S, C O I$ and per. Analysis was then conducted on the partitioned data sets (28S and per; $28 S$, per and COI) with the parameters as above.

When hybridization is involved, a single dichotomising phylogenetic tree will often not be a suitable representation of the phylogenetic history (Huson \& Bryant, 2006). This may make it necessary to use a more general graph, such as a network to represent the data. NeighborNet computes a set of splits from the data. If splits are compatible, the resultant graph will be a dichotomous tree, but when the splits are not compatible, it results in a network diagram with multiple parallel branches representing a single split (Huson \& Bryant, 2006). Network diagrams were created using NeighborNet in SplitsTree4 (Huson \& Bryant, 2006) using the uncorrected P-method for distance.

\section{RESULTS}

A total of 654 base pairs for $28 S, 576$ bp for $C O I$ and $722 \mathrm{bp}$ for per (a total of $1952 \mathrm{bp}$ ) were sequenced and aligned. There were no indels in the aligned sequences. A total of 77,83 and 76 specimens were sequenced respectively for 28S, COI and per (Table 3).

The ILD test showed $28 S$ and per to be congruent $(P=$ $0.99)$, and the ILD test for $28 S$ and $C O I$ was not statistically significant $(P=0.08)$. per and $C O I$ were significantly incongruent $(P=0.01)$ as was the combination of $28 S$, per and COI $(P=0.01)$. Due to the high level of congruence between $28 S$ and per, these two data sets were concatenated and used for the analyses and network diagrams. Despite the incongruence between the nuclear (28S and per) and mitochondrial (COI) data, these data sets were also concatenated and an analysis run on the total evidence.

The Bayesian Inference trees (Fig. 2A) for the nuclear genes ( $28 S$ and per) show both $L$. sericata and L. cuprina to be monophyletic clades with strong support (Fig. 2A). The Bayesian Inference tree for COI (Fig. 2B) shows that L. cuprina is paraphyletic with respect to $L$. sericata, with good posterior probability support. The first L. cuprina clade (Fig. 2B) exhibits both nuclear and mitochondrial sequences (and morphology) of "pure cuprina", while the second clade exhibits nuclear DNA (and morphology) of L. cuprina but mitochondrial DNA of L. sericata - a "hybrid" clade. The L. cuprina sequences from GenBank from Hawaii, Taiwan and China grouped with the "hybrid" clade (Fig. 2B).

Out of 42 specimens with the morphology of $L$. cuprina, five have mitochondrial genes that are typical of the L. sericata clade (Fig. 2B), but not of the "ancient hybrid" clade. The maximum parsimony trees were topologically compatible with the Bayesian Inference trees but the trees were less well resolved (trees not shown).

The network diagrams of the nuclear genes (28S and per) (Fig. 3) indicate a clear and simple split between the $L$. sericata specimens and the L. cuprina specimens. The COI network diagram (Fig. 4) shows two clear splits between a cluster of $L$. sericata specimens, and two clusters of $L$. cuprina specimens. The "hybrid" cluster of $L$. cuprina specimens lies closer to the L. sericata cluster than to the "pure" L. cuprina cluster, but is distinctively monophyletic. The five L. cuprina specimens that group 
TABLE 3. Specimen locality data for sequences from this study added to GenBank (* indicate identical sequences that are represented by one sequence in the Bayesian Inference tree, $\mathrm{M}$ - Male, $\mathrm{F}$ - Female)

\begin{tabular}{|c|c|c|c|c|c|c|}
\hline \multirow{2}{*}{ Species } & & & & & ession Numl & \\
\hline & Specimen & Loce & & $28 S$ & per & $\mathrm{COI}$ \\
\hline Calliphora vicina & CV_FRC_01 & Montferrier-Sur-Lez & France & JN792781 & & \\
\hline Lucilia caesar & Ca_FRC_01 & Montferrier-Sur-Lez & France & JN792782 & JN792858 & \\
\hline Lucilia infernalis & In_RWN_01 & Nyungwe Forest Reserve & Rwanda & JN792780 & JN792857 & JN813094 \\
\hline & C_AUS_01*(M) & Sydney & Australia & & & JN792622 \\
\hline & C AUS $02 *(\mathrm{~F})$ & Sydney & Australia & & & JN792623 \\
\hline & C_AUS_-03 (F) & Hornsby Heights & Australia & JN792705 & JN792783 & JN792624 \\
\hline & C_EGT_01 (F) & Alexandria & Egypt & JN792706 & JN792784 & JN792625 \\
\hline & C_EGT_02(F) & Alexandria & Egypt & JN792707 & JN792785 & JN792626 \\
\hline & C_SA_BFN_01(F) & Bloemfontein & South Africa & JN792708 & JN792786 & JN792627 \\
\hline & C_SA_BFN_02(F) & Bloemfontein & South Africa & JN792709 & JN792787 & JN792628 \\
\hline & C_SA_BRT_01 (F) & Britstown & South Africa & JN792710 & JN792788 & JN792629 \\
\hline & C_SA_BRT_02 (F) & Britstown & South Africa & JN792711 & JN792789 & JN792630 \\
\hline & C_SA_CT_01*(M) & Cape Town & South Africa & JN792712 & JN792790 & JN792631 \\
\hline & $\overline{\mathrm{C}} \mathrm{SA}^{-} \mathrm{CT}^{-} 02(\mathrm{~F})$ & Cape Town & South Africa & JN792713 & JN792791 & JN792632 \\
\hline & C_SA_CT_03*(F) & Cape Town & South Africa & JN792714 & JN792792 & JN792633 \\
\hline & C_SA_CT_04 (F) & Cape Town & South Africa & JN792715 & JN792793 & JN792634 \\
\hline & $\mathrm{C}^{-} \mathrm{SA}^{-} \mathrm{CT}^{-} 05(\mathrm{~F})$ & Cape Town & South Africa & JN792716 & JN792794 & JN792635 \\
\hline & C_SA_CT_06 (F) & Cape Town & South Africa & JN792717 & JN792795 & JN792636 \\
\hline & C_SA_CT_07 (F) & Cape Town & South Africa & JN792718 & JN792796 & JN792637 \\
\hline & C_SA_CT_08 (F) & Cape Town & South Africa & JN792719 & JN792797 & JN792638 \\
\hline & C_SA_CT_09*(F) & Cape Town & South Africa & JN792720 & JN792798 & JN792639 \\
\hline & C_SA_CT_10 (M) & Cape Town & South Africa & JN792721 & JN792799 & \\
\hline & C_SA_CT_11*(F) & Cape Town & South Africa & JN792722 & JN792800 & JN792640 \\
\hline & C_SA_CT_12*(F) & Cape Town & South Africa & JN792723 & JN792801 & JN792641 \\
\hline & C_SA_DBN_01*(F) & Durban & South Africa & JN792724 & JN792802 & JN792642 \\
\hline & C_SA_DBN_02 (F) & Durban & South Africa & JN792725 & JN792803 & JN792643 \\
\hline Lucilia cuprina & C_SA_DBN_03(M) & Durban & South Africa & JN792726 & JN792804 & JN792644 \\
\hline & C_SA_DBN_04 (F) & Durban & South Africa & & & JN792645 \\
\hline & C_SA_DBN_05 (F) & Durban & South Africa & & & JN792646 \\
\hline & C_SA_DBN_06 (F) & Durban & South Africa & JN792727 & JN792805 & JN792647 \\
\hline & C_SA_DBN_07*(F) & Durban & South Africa & JN792728 & JN792806 & JN792648 \\
\hline & C_SA_DBN_08 (F) & Durban & South Africa & JN792729 & JN792807 & JN792649 \\
\hline & C_SA_DBN_09 (F) & Durban & South Africa & JN792730 & JN792808 & JN792650 \\
\hline & C_SA_DBN_10*(F) & Durban & South Africa & JN792731 & JN792809 & JN792651 \\
\hline & C_SA_DBN_11*(F) & Durban & South Africa & JN792732 & JN792810 & JN792652 \\
\hline & C_SA_DBN_12 (F) & Durban & South Africa & JN792733 & JN792811 & JN792653 \\
\hline & C_SA_DBN_13 (F) & Durban & South Africa & JN792734 & JN792812 & JN792654 \\
\hline & C_SA_DBN_14*(F) & Durban & South Africa & JN792735 & JN792813 & JN792655 \\
\hline & C_SA_GHT_01(M) & Grahamstown & South Africa & JN792736 & JN792814 & JN792656 \\
\hline & C_SA_GHT_02 (F) & Grahamstown & South Africa & JN792737 & JN792815 & JN792657 \\
\hline & C_SA_NEL_01 (F) & Nelspruit & South Africa & JN792738 & JN792816 & JN792658 \\
\hline & C_SA_NEL_02 (F) & Nelspruit & South Africa & JN792739 & JN792817 & JN792659 \\
\hline & $\overline{\mathrm{C}} \_$THA_01 $(\mathrm{F})$ & Chiang Mai & Thailand & JN792740 & JN792818 & JN792660 \\
\hline & C_THA_02(F) & Chiang Mai & Thailand & JN792741 & JN792819 & JN792661 \\
\hline & C_THA_03 (F) & Chiang Mai & Thailand & JN792742 & JN792820 & JN792662 \\
\hline & C_THA_04 (F) & Chiang Mai & Thailand & & & JN792663 \\
\hline & C_USA_01 (F) & Merced & USA & JN792743 & JN792821 & JN792664 \\
\hline & C_USA_02(F) & Merced & USA & JN792744 & JN792822 & JN792665 \\
\hline & C_ZIM_01 (F) & Matobos & Zimbabwe & & & JN792666 \\
\hline & C_ZIM_02 (F) & Matobos & Zimbabwe & JN792745 & JN792823 & JN792667 \\
\hline & S_AUS_01 (M) & Seaford & Australia & JN792746 & JN792824 & JN792668 \\
\hline & S_CAN_01 (F) & Windsor & Canada & JN792747 & JN792825 & JN792669 \\
\hline & S_CAN_02 (F) & Windsor & Canada & JN792748 & JN792826 & JN792670 \\
\hline & S_FRC_01 (F) & Montferrier-Sur-Lez & France & JN792749 & JN792827 & JN792671 \\
\hline & S_FRC_02 (F) & Montferrier-Sur-Lez & France & JN792750 & JN792828 & JN792672 \\
\hline & S_FRC_03 (F) & Montferrier-Sur-Lez & France & JN792751 & JN792829 & JN792673 \\
\hline & S_GER_01 (F) & Kempen & Germany & JN792752 & & JN792674 \\
\hline & S_GER_02 (F) & Kempen & Germany & & JN792830 & JN792675 \\
\hline & S_GRC_01 (F) & Crete & Greece & JN792753 & & JN792676 \\
\hline & S_GRC_02 (F) & Crete & Greece & & & JN792677 \\
\hline & S_JPN_01*(F) & Osaka & Japan & JN792754 & JN792831 & JN792678 \\
\hline & S_JPN_02* (F) & Osaka & Japan & JN792755 & JN792832 & JN792679 \\
\hline & S_JPN_03* (F) & Iwate & Japan & JN792756 & JN792833 & JN792680 \\
\hline & S_JPN_04*(F) & Iwate & Japan & JN792757 & JN792834 & JN792681 \\
\hline & S_NAM_01 (F) & Possession Island & Namibia & JN792758 & JN792835 & JN792682 \\
\hline & S_NAM_02 (F) & Possession Island & Namibia & JN792759 & JN792836 & JN792683 \\
\hline & S_SA_CT_01* (F) & Cape Town & South Africa & JN792760 & JN792837 & JN792684 \\
\hline & S_SA_CT_02 (F) & Cape Town & South Africa & JN792761 & JN792838 & JN792685 \\
\hline Lucilia sericata & S_SA_CT_03*(M) & Cape Town & South Africa & JN792762 & JN792839 & JN792686 \\
\hline & S_SA_CT_04*(F) & Cape Town & South Africa & JN792763 & JN792840 & JN792687 \\
\hline & S_SA_CT_05 (F) & Cape Town & South Africa & JN792764 & JN792841 & JN792688 \\
\hline & S_SA_CT_06*(F) & Cape Town & South Africa & JN792765 & JN792842 & JN792689 \\
\hline & S_SA_CT_07* (F) & Cape Town & South Africa & JN792766 & JN792843 & JN792690 \\
\hline & S_SA_CT_08*(F) & Cape Town & South Africa & JN792767 & JN792844 & JN792691 \\
\hline & S_SA_GHT_01 (F) & Grahamstown & South Africa & JN792768 & JN792845 & JN792692 \\
\hline & S_SA_GHT_02 (F) & Grahamstown & South Africa & JN792769 & JN792846 & JN792693 \\
\hline & S_SA_PTA_01 (M) & Pretoria & South Africa & JN792770 & JN792847 & JN792694 \\
\hline & S_SA_PTA_02 (F) & Pretoria & South Africa & JN792771 & JN792848 & JN792695 \\
\hline & S_SA_PTA_03 (F) & Pretoria & South Africa & JN792772 & JN792849 & JN792696 \\
\hline & S_SA_PTA_04 (M) & Pretoria & South Africa & JN792773 & JN792850 & JN792697 \\
\hline & S_SA_WLK_01 (F) & Welkom & South Africa & JN792774 & JN792851 & JN792698 \\
\hline & S_SA_WLK_02 (F) & Welkom & South Africa & JN792775 & JN792852 & JN792699 \\
\hline & S_SA_WTB_01 (F) & Witbank & South Africa & JN792776 & JN792853 & JN792700 \\
\hline & S_SA_WTB_02 (F) & Witbank & South Africa & JN792777 & JN792854 & JN792701 \\
\hline & S_SWZ_01 (M) & Lausanne & Switzerland & & & JN792702 \\
\hline & S_USA_01 (F) & Michigan & USA & JN792778 & JN792855 & JN792703 \\
\hline & S_USA_02 (M) & Michigan & USA & JN792779 & JN792856 & JN792704 \\
\hline
\end{tabular}




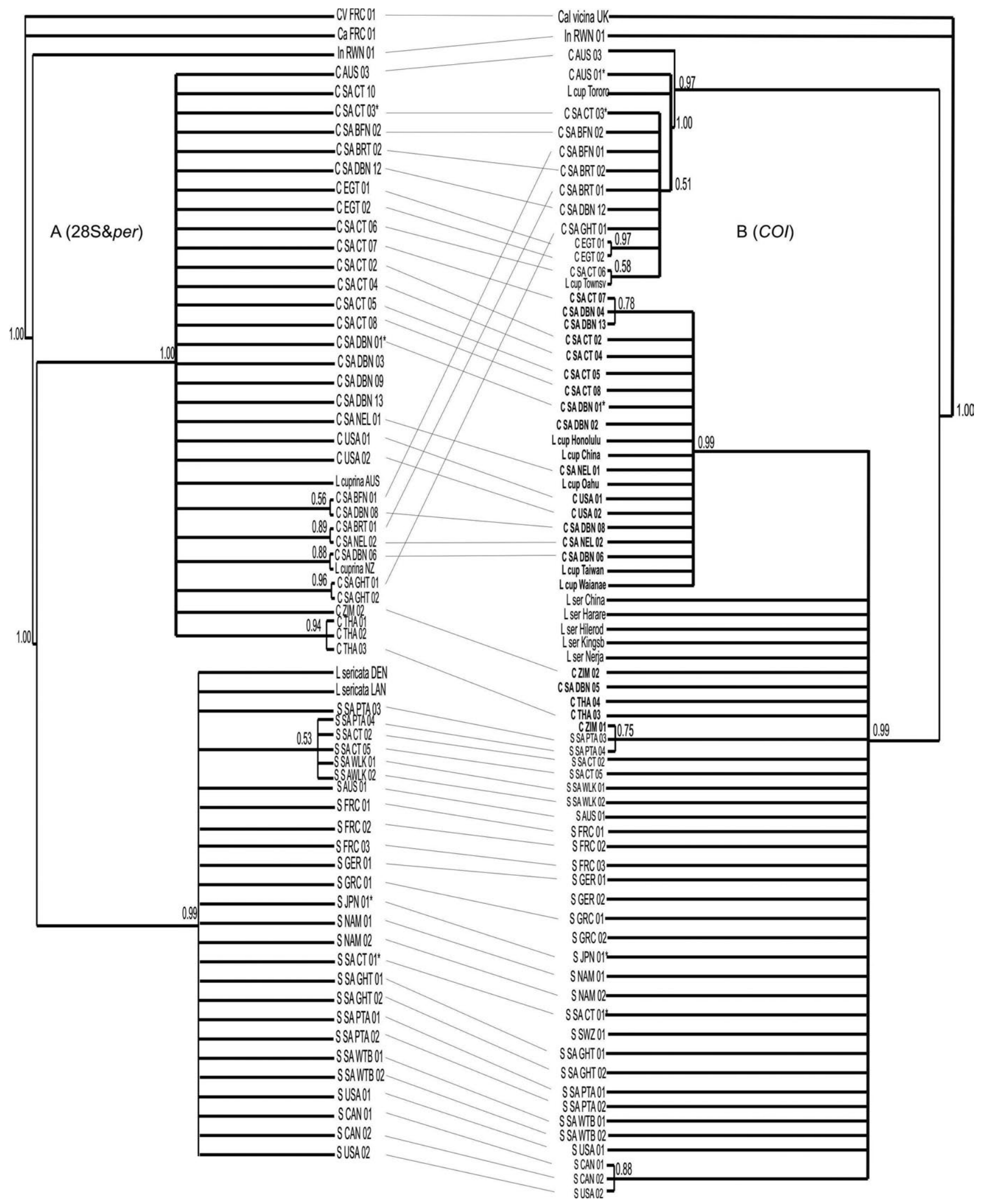

Fig. 2. Bayesian Inference trees constructed from nuclear genes (28S and per) (A) and mitochondrial genes (COI) (B) data. Posterior probabilities are indicated on nodes. C - cuprina, $\mathrm{S}$ - sericata, $\mathrm{CV}$ - Calliphora vicina, $\mathrm{In}$ - Lucilia infernalis, CA - Lucilia caesar, AUS - Australia, CAN - Canada, FRC - France, GER - Germany, GRC - Greece, JPN - Japan, NAM - Namibia, EGT Egypt, RWN - Rwanda, SWZ - Switzerland, SA - South Africa, THA - Thailand, USA - United States of America, ZIM - Zimbabwe, CT - Cape Town, BFN - Bloemfontein, BRT - Britstown, DBN - Durban, GHT - Grahamstown, NEL - Nelspruit, PTA Pretoria, WLK - Welkom, WTB - Witbank. 


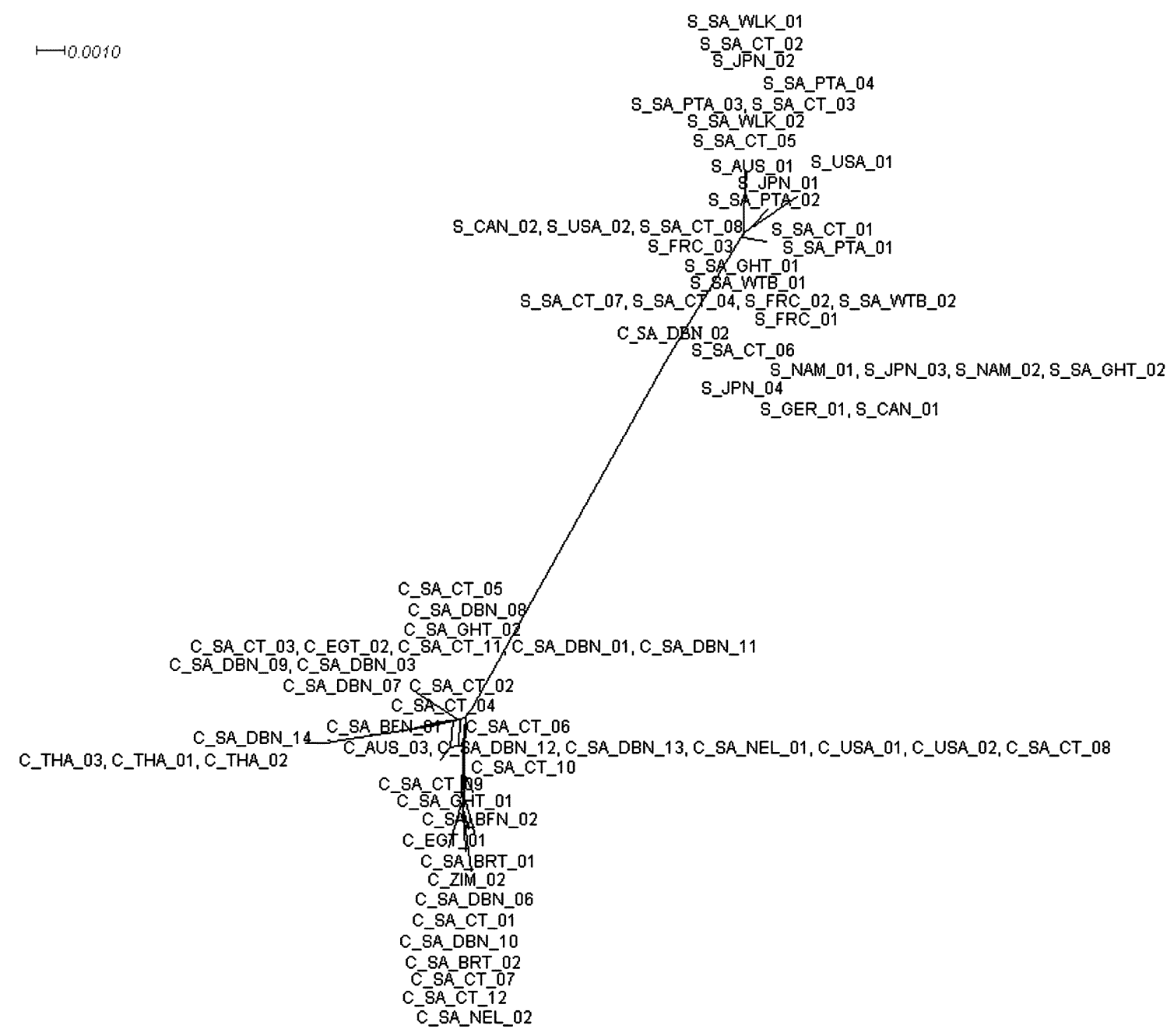

Fig. 3. NeighborNet network diagram constructed from $28 S$ \& per data. C - cuprina, S - sericata, AUS - Australia, CAN - Canada, FRC - France, GER - Germany, JPN - Japan, NAM - Namibia, EGT - Egypt, SA - South Africa, THA - Thailand, USA United States of America, ZIM - Zimbabwe, CT - Cape Town, BFN - Bloemfontein, BRT - Britstown, DBN - Durban, GHT Grahamstown, NEL - Nelspruit, PTA - Pretoria, WLK - Welkom, WTB - Witbank.

within the L. sericata clade (Fig. 2B) also appear within the L. sericata cluster (Fig. 4). The network diagram of the total evidence concatenated data sets (Fig. 5) shows a clear split between the L. sericata and L. cuprina clusters, and the L. cuprina samples split into two clusters which are linked by more pathways to each other than to the $L$. sericata cluster.

\section{DISCUSSION}

A number of studies have been conducted on L. sericata and L. cuprina, looking at morphological identification, the possibility that they are interbreeding and whether L. cuprina should be classified as two subspecies or two independent species (Ullyett, 1945; Waterhouse \& Paramonov, 1950; Norris, 1990; Holloway, 1991a, b; Stevens \& Wall, 1996; Stevens et al., 2002; Stevens, 2003; Wallman et al., 2005; Wells et al., 2007; Harvey et al., 2008; Tourle et al., 2009; DeBry et al., 2010). This study focuses on these two species in South Africa, but also examines specimens from across the globe to place the South African situation into a global context. This study used two nuclear and one mitochondrial gene where most previous studies have either used only one mitochondrial gene or a combination of mitochondrial genes and one nuclear gene (Table 1). Stevens \& Wall (1996) used RAPDs, which encompasses multi-locus nuclear genotype data, but without targeting explicit genes (Table 1).

Individually and together, the nuclear $28 S$ and per genes show L. sericata and L. cuprina to be two monophyletic clades (Fig. 2A) with very strong posterior probability support (0.99 and 1.00 respectively). However, the mitochondrial COI gene suggests that $L$. cuprina is paraphyletic with respect to $L$. sericata (Fig. 2B). There is a monophyletic clade of $L$. cuprina specimens that have $L$. sericata-like mtDNA, which has been seen in previous studies (Table 1). This monophyletic clade of L. cuprina with $L$. sericata-like mtDNA has been suggested to represent an ancient hybridization event (Stevens \& Wall, 1996; Stevens et al., 2002; Tourle et al., 2009). The $L$. sericata mtDNA appears to have been fixed in this lineage of $L$. cuprina and not lost through lineage sorting.

However, there are also five specimens with the morphology of L. cuprina and mtDNA of L. sericata that are not representative of the ancient, introgressed clade (Figs 


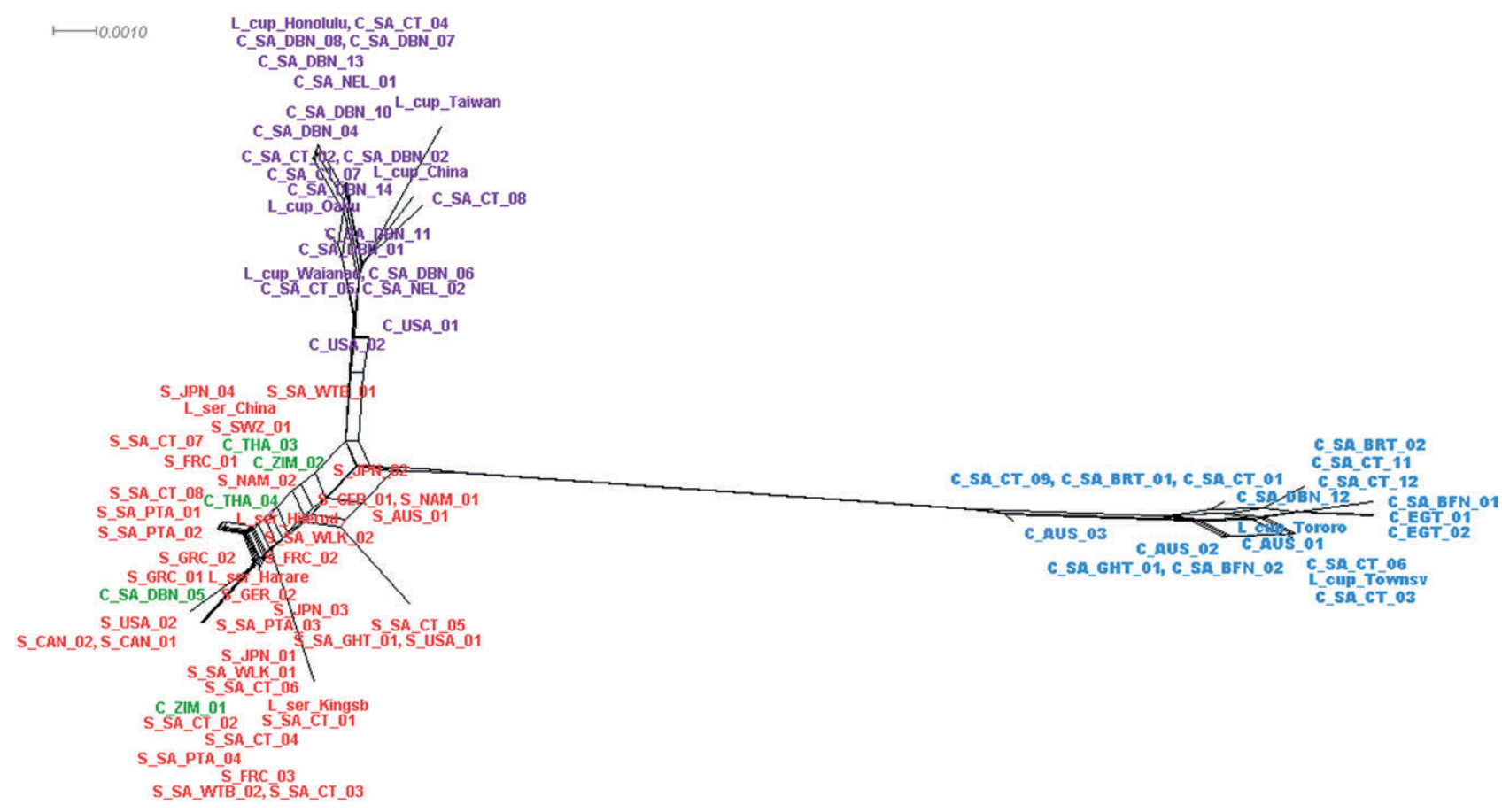

Fig. 4. NeighborNet network diagram constructed from COI data. C - cuprina, $\mathrm{S}$ - sericata, AUS - Australia, CAN - Canada, FRC - France, GER - Germany, JPN - Japan, NAM - Namibia, EGT - Egypt, SA - South Africa, THA - Thailand, USA - United States of America, ZIM - Zimbabwe, CT - Cape Town, BFN - Bloemfontein, BRT - Britstown, DBN - Durban, GHT - Grahamstown, NEL - Nelspruit, PTA - Pretoria, WLK - Welkom, WTB - Witbank.

2B and 4), implying novel mismatches of nuclear and mitochondrial genomes. Nuclear genes were not amplified for three of these specimens, but the other two, from Zimbabwe and Thailand, have (different) $28 S$ and per genotypes typical of L. cuprina, which suggests modern hybridization. This has not been seen in any previous studies on L. sericata / L. cuprina (Table 1) and provides the first direct genetic evidence of modern-day natural interbreeding between these species.

\section{Ancient hybrids and introgression}

The specimens that form the monophyletic clade of $L$. cuprina with L. sericata-like mtDNA originate from Durban, Nelspruit and Cape Town in South Africa, and from Merced in California in the continental USA, Hawaii, China, and Taiwan (Tables 2 and 3). It was once suggested that this lineage was restricted to the Hawaiian Islands (Stevens \& Wall, 1996; Stevens et al., 2002), but since then the lineage has been found in North America, Africa, and Asia. It would be difficult to determine where it originated because it is so widespread. There does not appear to be any geographical coherence within the two L. cuprina clades (Fig. 2B). It was suggested that the two named subspecies of $L$. cuprina $-L$. c. cuprina and $L$. c . dorsalis - could be distinguished using COI sequences because both subspecies formed monophyletic clades (DeBry et al., 2010), with L. c. cuprina forming a monophyletic clade that was sister to the L. sericata clade, thus suggesting that all $L$. cuprina with $L$. sericata-like mtDNA are L. c. cuprina. Sequences from South Africa (Tourle et al., 2009) that were included in this analysis (DeBry et al., 2010) all grouped with the putative clade of
L. c. cuprina, although African L. cuprina are considered to be L. cuprina dorsalis (Waterhouse \& Paramonov, 1950). Perhaps L. c. cuprina has been introduced into South Africa like some other synanthropic blow flies (Williams \& Villet, 2006), but the problem remains of distinguishing them morphologically, an issue that was addressed by Tourle et al. (2009), who found the "hybrid" clade to have a morphological index that was more cuprina-like than "pure" cuprina specimens.

Four cases of mtDNA introgression without detectable nuclear introgression, as seen in this study, were reported for Protocalliphora blowflies (Whitworth et al., 2007). Interspecific mitochondrial introgression linked to selective sweeps induced by nuclear-cytoplasmic incompatibility due to Wolbachia infections has been described in various insects (Ballard, 2000) as an explanation for how mtDNA introgression without nuclear introgression is possible. Cytoplasmic incompatibility is a process where, if uninfected females mate with infected males, some or all of their eggs will die. But if an infected female mates with either an infected or uninfected male, her eggs remain viable but all will be infected with Wolbachia. So infected females outcompete uninfected ones and the overall population of Wolbachia-infected flies (and therefore Wolbachia) increases (Zimmer, 2001). Thus the mitochondria of infected individuals have a greater chance than uninfected individuals of being passed on because mitochondria are passed down the female line, leading to fixed introgression. Wolbachia infection in the blowfly Protocalliphora sialia (Baudry et al., 2003) and infections of three different strains of Wolbachia in Protocalliphora in North America (Whitworth et al., 2007) 


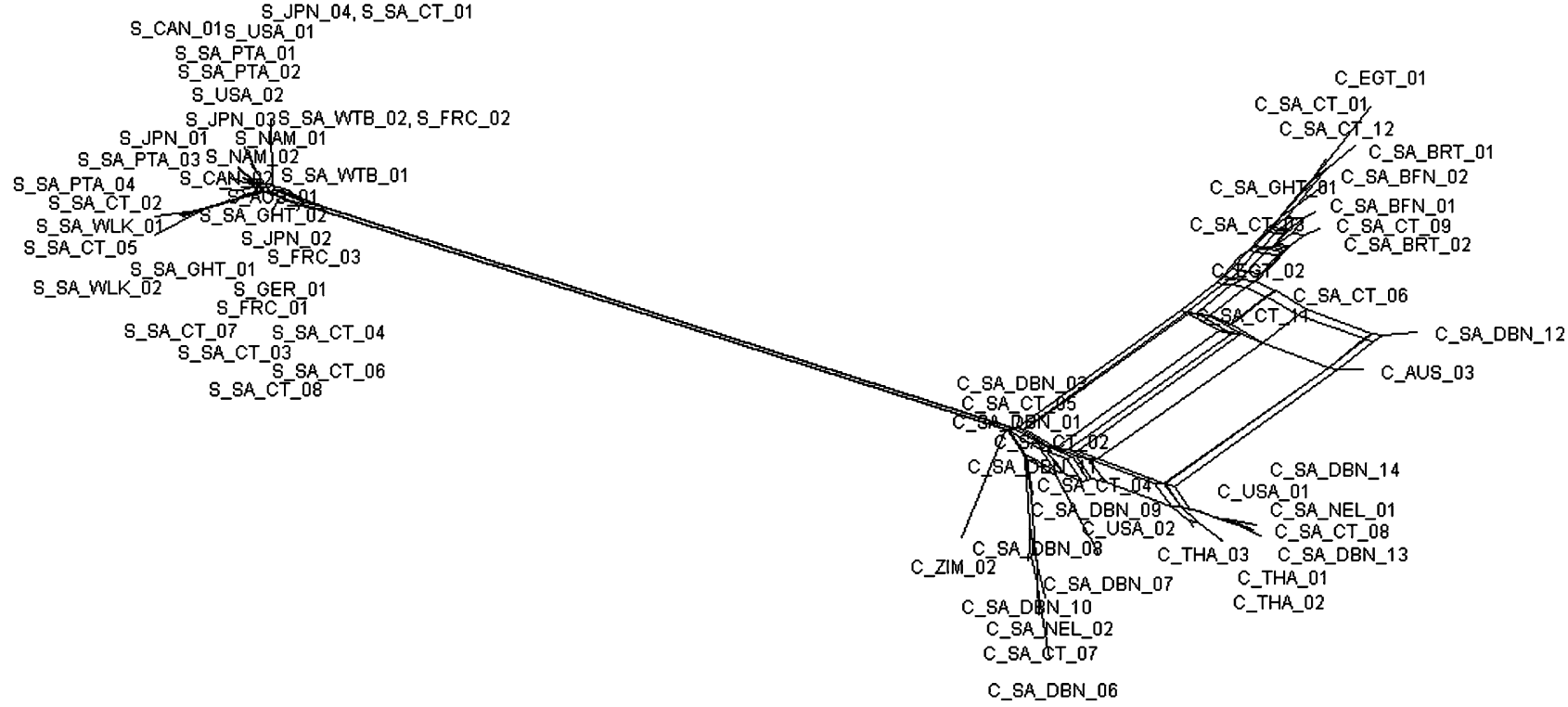

Fig. 5. NeighborNet network diagram constructed from $28 S \&$ per \& COI concatenated data. C - cuprina, S - sericata, AUS Australia, CAN - Canada, FRC - France, GER - Germany, JPN - Japan, NAM - Namibia, EGT - Egypt, SA - South Africa, THA - Thailand, USA - United States of America, ZIM - Zimbabwe, CT - Cape Town, BFN - Bloemfontein, BRT - Britstown, DBN Durban, GHT - Grahamstown, NEL - Nelspruit, PTA - Pretoria, WLK - Welkom, WTB - Witbank.

have been reported. All of these infections resulted in mtDNA introgression without any detectable nuclear introgression. Further studies are recommended to determine if Lucilia blowflies are affected by Wolbachia infections as an explanation for the pattern seen in this study. However, such infections can die out over time, so that the only evidence of them may be cytoplasmic introgression (Zimmer, 2001).

The combined $28 S$ and per data show a very clear split between the L. sericata and L. cuprina samples (Fig. 3). The splits show very little internal incompatibility. The mtDNA (COI) shows a much higher degree of incompatibility between the splits (Fig. 4) which represents incompatible signals (Huson \& Bryant, 2006). There are three important splits that group $L$. sericata together and two $L$. cuprina splits. This grouping is consistent with the Bayesian Inference tree (Fig. 2B). The concatenated total data set (28S, per and COI) (Fig. 5) shows a high level of incompatibility between the $L$. cuprina samples and a high degree of compatibility between the L. sericata samples. The L. cuprina samples show a number of splits and this incompatibility is probably as a result of the $L$. sericata-like mitochondrial DNA which results in the two clusters of L. cuprina.

\section{Modern hybrids}

The genetic component of an organism's morphology is determined by its nuclear DNA. One would expect recombination of the nuclear DNA if interbreeding occurs, resulting in morphology that is either intermediate (for multi-locus traits) or a mosaic of the two parental phenotypes (for single-locus traits). However, if one species' alleles are consistently dominant over the other, then despite recombination, the dominant phenotype will prevail (Lewin, 1997). Thus, although the putative modern hybrids had sericata-like mtDNA indicating hybridisation, they were still L. cuprina-like in morphology, suggesting that L. cuprina's alleles for morphology are dominant over those of $L$ sericata. In crossing experiments carried out in a laboratory, it was suggested that the femur colour of $L$. cuprina and the abdomen colour of $L$. sericata were dominant characteristics, giving the hybrids a combination of the two species' morphologies (Ullyett, 1945). However, this study used only two characters (femur and abdomen colour) which Ullyett (1945) described as not being "scientific criteria" because there are gradations in both characters depending on both the age and condition of the specimens and the observers' opinion and thus they could not be considered reliable criteria for identification.

Even when hybridization occurred in Hyalomma (Acari: Ixodidae), no intermediate morphologies were observed and the morphology of one parent appeared to be inherited over that of the other (Rees et al., 2003). Funk \& Omland (2003) suggest that most hybrid species originate via asymmetrical hybridization and would be mitochondrially monophyletic. This might explain what we see in this study regarding the ancient hybridization "hybrid" group, but not the modern hybrids (which are derived from several sources). mtDNA may be more susceptible to introgression than nuclear loci (Machado \& Hey, 2003). One is therefore less likely to have consistent gene trees for mtDNA and they may even suggest a different phylogeny. This gives support to the wellestablished idea that more than just one nuclear or 
mitochondrial gene needs to be used when trying to determine species and gene trees (Funk \& Omland, 2003; Machado \& Hey, 2003; Hurst \& Jiggins, 2005).

\section{DNA-based identification}

The use of COI sequences to correctly identify the two presumed subspecies of $L$. cuprina seems unlikely to succeed due to the presence of L. cuprina flies that group within the L. sericata clade (Fig. 2B). The phylogenetic positioning of these flies indicates their relationship relative to other specimens, but does not necessarily give an identification that agrees with their morphology. This problem is even more acute for modern hybrids. It also raises the issue of using $C O I$ as the universal "barcoding" gene and whether it is suitable, especially for insects (Rubinoff et al., 2006; Roe \& Sperling, 2007; Whitworth et al., 2007; Jordaens et al., 2012; Sonet et al., 2012). The idea of using part of $C O I$ as a universal diagnostic gene is to allow the identification of unknown specimens when comparing them to identified species' sequences (Roe \& Sperling, 2007). However, using COI alone could result in incorrect identifications, as seen in this study, as numerous insect species have undergone hybridisation and may carry mtDNA of another species (Zimmer, 2001; Baudry et al., 2003; Whitworth et al., 2007). The sequences of unidentified specimens may align with species with which they share mtDNA, but which are in fact a different species based on nuclear DNA or morphology. Although a study on blowflies in Australia suggested that using COI for identification is tenable, the authors also raised the issue of misidentifications when hybridisation was involved and suggested the use of a nuclear gene for confirmation (Nelson et al., 2007). A study of 1333 mitochondrial sequences (minimum of $300 \mathrm{bp}$ ) for 449 species of flies concluded that using $\mathrm{COI}$ alone for identification had a less than $70 \%$ success rate at identifying the species correctly (Meier et al., 2006).

The results show that in some cases both nuclear and mitochondrial genes are needed for reliable species identification and hybrid detection. It is well known that the use of just one gene can generally be taxonomically misleading as can be seen in the L. sericata / L c cuprina situation (Wallman et al., 2005; Harvey et al., 2008; Tourle et al., 2009; DeBry et al., 2010), especially if modern hybridisation is occurring at any appreciable rate. By using nuclear genes in conjunction with mitochondrial genes, a potentially misleading situation can be avoided (Rubinoff et al., 2006; Nelson et al., 2007; Roe \& Sperling, 2007; Williams et al., 2008; Tantawi et al., 2010).

ACKNOWLEDGEMENTS. We thank F. Cronje, D. Emery, G. Goergen, C. Hänel, O. Harrison, C. Kelly, A. Kirk-Spriggs, N. Lunt, M. Mansell, R. Merrit, N. Mkize, H. Numata, C. Richards, K. Saigusa, K. Sink, K. Smith, K. Sukontason, J. Wallman, G. Whittington-Jones, and C. Wyss for providing specimens from different localities; O. Harrison for generating the locality map; J. Lamb (University of KwaZulu-Natal) for access to facilities; J. Vivian and T. Naidoo for laboratory assistance and advice; T. Pape and two anonymous referees for their valuable comments on the draft manuscript; and the Durban Natural Science Museum, the National Research Foundation of South Africa and
Rhodes University for financial support. Any opinion, findings and conclusions or recommendations expressed in this material are those of the authors and do not necessarily reflect the views of the National Research Foundation.

\section{REFERENCES}

AltinciceK B. \& Vilcinskas A. 2009: Septic injury-inducible genes in medicinal maggots of the green blow fly Lucilia sericata. - Insect Mol. Biol. 18: 119-125.

Aubertin D. 1933: Revision of the genus Lucilia R.-D. (Diptera, Calliphoridae). - J. Linn. Soc. Lond. (Zool.) 38: 389-463.

BALLARD J.W.O. 2000: When one in not enough: Introgression of mitochondrial DNA in Drosophila. - Mol. Biol. Evol. 17: 1126-1130.

Baudry E., Bartos J., Emerson K., Whitworth T. \& Werren H. 2003: Wolbachia and genetic variability in the birdnest blowfly Protocalliphora sialia. - Mol. Ecol. 12: 1843-1854.

CRAGG J.B. 1956: The olfactory behaviour of Lucilia species (Diptera) under natural conditions. - Ann. Appl. Biol. 44: 467-477.

Crombe A.C. 1944: On the measurement and modification of the olfactory responses of blowflies. - J. Exp. Biol. 120: 159-166.

DeBry R., Timm A.E., Dahlem G.A. \& Stamper T. 2010: mtDNA-based identification of Lucilia cuprina (Wiedemann) and Lucilia sericata (Meigen) (Diptera: Calliphoridae) in the continental United States. - Forensic Sci. Int. 202: 102-109.

Farris J.S., Källersjö M., Kluge A.G. \& Bult C. 1994: Testing significance of congruence. - Cladistics 10: 315-319.

FunK D.J. \& OMLand K.E. 2003: Species-level paraphyly and polyphyly: Frequency, causes and consequences, with insights from animal mitochondrial DNA. - Annu. Rev. Ecol. Evol. Syst. 34: 397-423.

Hall T.A. 1999: BioEdit: a user-friendly biological sequence alignment editor and analysis programme for Windows 95/98/NT. - Nucl. Acid Symp. Ser. 41: 95-98.

Harvey M.L., Gaudieri S., Villet M.H. \& Dadour I.R. 2008: A global study of forensically significant calliphorids: Implications for identification. - Forensic Sci. Int. 177: 66-76.

Heath A.C.G. \& Bishop D.M. 2006: Flystrike in New Zealand: An overview based on a 16-year study, following the introduction and dispersal of the Australian sheep blowfly, Lucilia cuprina Wiedemann (Diptera: Calliphoridae). - Vet. Parisitol. 137: 333-344.

Hepburn G.A. 1943: Sheep blowfly research I - A survey of maggot collections from live sheep and a note on the trapping of blowflies. - Onderstepoort J. Vet. 18: 13-18.

Holloway B.A. 1991a: Morphological characters to identify adult Lucilia sericata (Meigen, 1826) and L. cuprina (Wiedemann, 1830) (Diptera: Calliphoridae). - N. Z. J. Zool. 18: 415-420.

Holloway B.A. 1991b: Identification of third-instar larvae of flystrike and carrion-associated blowflies in New Zealand (Diptera: Calliphoridae). - N. Z. Entomol. 14: 24-28.

Hurst G.D.D. \& Jiggins F.M. 2005: Problems with mitochondrial DNA as a marker in population, phylogeographic and phylogenetic studies: the effects of inherited symbionts. Proc. R. Soc. (B) 272: 1525-1534.

Huson D.H. \& BRYANT D. 2006: Application of phylogenetic networks in evolutionary studies. - Mol. Biol. Evol. 23: 254-267.

Jordaens K., Sonet G., Richet R., Dupont E., Braet Y. \& Desmyter S. 2012: Identification of forensically important Sarcophaga species (Diptera: Sarcophagidae) using the mito- 
chondrial COI gene. - Int. J. Legal Med. (in press) DOI: 10.1007/s00414-012-0767-6.

Kingu H.J.C., Kuria S.K., Villet M.H., Mkhize J.N., Dhaffala A. \& IISA J.M. 2012: Cutaneous myiasis: is Lucilia cuprina safe and acceptable for maggot debridement therapy? $-J$. Cosmet. Dermatol. Sci. Appl. 2: 79-82.

Lewin B. 1997: Genes VI. Oxford University Press, New York, $1260 \mathrm{pp}$.

LOUw S.v.D.M. \& VAN DER LINDE T.C. 1993: Insects frequenting decomposing corpses in central South Africa. - Afr. Entomol. 1: 265-269.

Machado C.A. \& Hey J. 2003: The causes of phylogenetic conflict in a classic Drosophila species group. - Proc. R. Soc. (B) 270: 1193-1202.

Meier R., Shiyang K., Vaidya G. \& NG P.K.L. 2006: DNA barcoding and taxonomy in Diptera: A tale of high intraspecific variability and low identification success. - Syst. Biol. 55: $715-728$

Nelson L.A., Wallman J.F. \& Dowton M. 2007: Using COI barcodes to identify forensically and medically important blowflies. - Med. Vet. Entomol. 21: 44-52.

Nichols R. 2001: Gene trees and species trees are not the same. — Trends Ecol. Evol. 16: 358-364.

NoRRIS K.R. 1990: Evidence for the multiple exotic origin of Australian populations of the sheep blowfly, Lucilia cuprina (Wiedemann) (Diptera: Calliphoridae). - Aust. J. Zool. 38: 635-648.

NuIn P. 2005: MrMTgui 1.0 (Version 1.6). Program distributed by the author at http://www.genedrift.org/mtgui.php

Nylander J.A.A. 2004: MrModeltest v2. Program distributed by the author. Evolutionary Biology Centre, Uppsala University.

Page R.D.M. \& Charleston M.A. 1997: From gene to organismal phylogeny: reconciled trees and the gene tree/species tree problem. - Mol. Phylogenet. Evol. 7: 231-240.

Paul A.G., Ahmad N.W., Lee H.L., Ariff A.M., Saranum M., Naicker A.S. \& Osman Z. 2009: Maggot debridement therapy with Lucilia cuprina: a comparison with conventional debridement in diabetic foot ulcers. - Int. Wound J. 6: $39-46$.

Rees D.J., Dioli M. \& Kirkendall L.R. 2003: Molecules and morphology: evidence for cryptic hybridization in African Hyalomma (Acari: Ixodidae). - Mol. Phylogenet. Evol. 27: 131-142.

Rubinoff D., Cameron S. \& Will K. 2006: A genomic perspective on the shortcomings of mitochondrial DNA for "barcoding" identification. - J. Hered. 97: 581-594.

Simon C., Buckley T.R., Frati F., Stewart J.B. \& Beckenbach A.T. 2006: Incorporating molecular evolution into phylogenetic analysis, and a new compilation of conserved polymerase chain reaction primers for animal mitochondrial DNA. - Annu. Rev. Ecol. Evol. Syst. 37: 545-579.

Sмiтн K.E. 1986: A Manual of Forensic Entomology. British National History Museum, London, $205 \mathrm{pp}$.

Sonet G., Jordaens K., Braet Y. \& Desmyter S. 2012: Why is the molecular identification of the forensically important blowfly species Lucilia caesar and L. illustris (family Calliphoridae) so problematic? - Forensic Sci. Int. 223: 153-159.

SperLing F.A.H., ANDERson G.S. \& Hickey D.A. 1994: A DNAbased approach to the identification of insect species used for postmortem interval estimation. - J. Forensic Sci. 39: 418-427.

Stevens J.R. 2003: The evolution of myiasis in blowflies (Calliphoridae). - Int. J. Parasitol. 33: 1105-1113.

Stevens J. \& Wall R. 1996: Species, sub-species and hybrid populations of the blowflies Lucilia cuprina and Lucilia sericata (Diptera: Calliphoridae) — Proc. Biol. Sci. 263: 1335-1341.

Stevens J.R., Wall R. \& Wells J.D. 2002: Paraphyly in Hawaiian hybrid blowfly populations and the evolutionary history of anthropophilic species. - Insect Mol. Biol. 11: 141-148.

Swofford D.L. 2003: PAUP* Phylogenetic Analysis Using Parsimony (*and Other Methods), Version 4. Sinauer Associates, Sunderland, MA.

Tantawi T.I., Williams K.A. \& Villet M.H. 2010: An accidental but safe and effective use of Lucilia cuprina (Diptera: Calliphoridae) in maggot debridement therapy in Alexandria, Egypt. - J. Med. Entomol. 47: 491-494.

Tourle R., Downie D.A. \& Villet M.H. 2009: A morphological and molecular comparison of Lucilia cuprina and L. sericata (Diptera: Calliphoridae) in South Africa. - Med. Vet. Entomol. 23: 6-14.

UlLyett G.C. 1945: Species of Lucilia attacking sheep in South Africa. - Nature 155: 636-637.

VILCINSKAS A. 2011: From traditional maggot therapy to modern biosurgery. In Vilcinskas A. (ed.): Insect Biotechnology. Springer, Dordrecht, pp. 67-75.

Vogt W.G. \& Woodburn T.L. 1979: Ecology, distribution and importance of sheep myiasis flies in Australia. In: National Symposium of the Sheep Blowfly and Flystrike in Sheep. N.S.W. Dep. Agric., Sydney, pp. 23-32.

Wallman J.F., Leys R. \& Hogendoorn K. 2005: Molecular systematics of Australian carrion-breeding blowflies (Diptera: Calliphoridae) based on mitochondrial DNA. - Invertebr. Syst. 19: 1-15.

Waterhouse D.F. \& Paramonov S.J. 1950: The status of the two species of Lucilia (Diptera, Calliphoridae) attacking sheep in Australia. - Aust. J. Sci. Res. 3: 310-336.

Wells J.D., Wall R. \& Stevens J.R. 2007: Phylogenetic analysis of forensically important Lucilia flies based on cytochrome oxidase I sequence: a cautionary tale for forensic species determination. - Int. J. Legal Med. 121: 229-233.

Whitworth T.L., Dawson R.D., Magalon H. \& Baudry E. 2007: DNA barcoding cannot reliably identify species of the blowfly genus Protocalliphora (Diptera: Calliphoridae). Proc. R. Soc. (B) 274: 1731-1739.

Williams K.A. \& Villet M.H. 2006: A new and earlier record of Chrysomya megacephala in South Africa, with notes on another exotic species, Calliphora vicina (Diptera: Calliphoridae). - Afr. Invertebr. 47: 347-350.

Williams K.A., Cronje F.J., Avenant L. \& Villet M.H. 2008: Identifying flies used for maggot debridement therapy. $-S$. Afr. Med. J. 98: 196-197.

ZimMER C. 2001: Wolbachia: A tale of sex and survival. - Science 292: 1093-1095.

Received May 31, 2012; revised and accepted November 19, 2012 